Valerie Heffernan

\title{
Unschweizerische Schweizerliteratur?
}

\author{
Ruth Schweikert, Peter Stamm, Zoë Jenny
}

In einem 2003 erschienenen Band zur aktuellen Lage der Schweizer Literatur diagnostizierte FAZ-Kritikerin Pia Reinacher »eine[n] tief greifenden Wandel« in der jüngsten Schweizer Literatur. ${ }^{1}$ Die neueren Schweizer Autoren - die teilweise erstaunlich hohe Verkaufszahlen erreichten ${ }^{2}$ - setzen sich, so Reinacher, entschieden von ihren literarischen und schriftstellerischen Vorfahren ab:

Thema dieser jüngeren Schweizer Autorinnen und Autoren ist nicht mehr die Schweiz. Ihre Träume verheddern sich nicht mehr in der guten oder bösen Heimat. Das literarische Herz dieser nachrückenden Generation schlägt weder für noch gegen das Vaterland, sondern vielmehr für die eigene Biographie, für Liebe, Sex und Partnerstress. Zoë Jenny, Peter Weber, Ruth Schweikert, Perikles Monioudis, Aglaja Veteranyi, Peter Stamm, Raphael Urweider oder Silvio Huonder wenden sich in ihren Büchern gelassen von den Vätern ab und gehen eigene Wege. ${ }^{3}$

Reinachers Beobachtungen werfen wichtige Fragen bezüglich des Selbstverständnisses der jüngeren Schweizer Schriftstellergeneration auf. Sie deuten auf einen grundlegenden Bruch mit dem Konzept einer littérature engagée hin, wie sie von Max Frisch, Friedrich Dürrenmatt, Walter Matthias Diggelmann, Peter Bichsel, Adolf Muschg, Hugo Loetscher und anderen Schweizern geschrieben wurde. Anders als die politisch und sozial engagierten Schriftsteller der 1960er und 1970er Jahre schenken die Angehörigen dieser Generation der politischen und sozialen Lage der Schweiz viel weniger Aufmerksamkeit; die Schweizer Identität hinterlässt daher, so Reinacher, »immer geringere Spuren im Text «. ${ }^{4}$ Vielmehr richtet sich ihr Blick nach außen und auf Motive und Probleme, die allgemein relevant sind und dadurch eine breitere Leserschaft ansprechen. Reinacher fällt auf, dass die neueren Schriftsteller die traditionellen Anliegen der Schweizer Literatur hinter sich gelassen haben, daher kommt sie zum uneingeschränkten Schluss, dass sie »der Heimat und ihren Stoffen entlaufen sind «. ${ }^{5}$

Reinachers Darstellung der literarischen Situation der Schweiz am Anfang des 21. Jahrhunderts tendiert zweifellos zur Verallgemeinerung und Vereinfachung, doch spiegelt sie zumindest teilweise Max Frischs fast vierzig Jahre zuvor

1 Pia Reinacher: Je Suisse. Zur aktuellen Lage der Schweizer Literatur. München, Wien: Nagel \& Kimche 2003, S. 9.

2 In dieser Hinsicht sei vor allem auf den Verkaufserfolg von Zoë Jennys und Peter Stamms Romanen der späten 1990er Jahre hingewiesen, sowohl innerhalb der Schweiz als auch auf dem internationalen Markt.

3 Reinacher: Je Suisse (Anm. 1), S. 9.

4 Ebd., S. 44.

5 Ebd., S. 21.

Please cite as: Valerie Heffernan, 'Unschweizerische Schweizerliteratuer? Ruth Schweikert, Peter Stamm, Zoë Jenny’, in: Schweiz schreiben. Zu Konstruktion und Dekonstruktion des Mythos Schweiz in der Gegenwartsliteratur, ed. by Jürgen Barkhoff and V. Heffernan, Tübingen: De Gruyter, 2010, pp. 283-295. 
artikulierte Auseinandersetzung mit der jüngeren Literatur seiner Zeit wider. In seinem 1965 verfassten Essay Unbewältigte schweizerische Vergangenheit macht Frisch seinen jüngeren Kollegen den Vorwurf, sich nicht ausreichend mit ihrer Heimat auseinandergesetzt zu haben und vor allem dem heiklen Thema ihres zweifelhaften Verhaltens während des Zweiten Weltkriegs ausgewichen zu sein. ${ }^{6}$ Frisch bedauert, dass die jüngere Literatur der Schweiz »fast ausnahmslos apolitisch oder abstrakt-politisch « sei, ${ }^{7}$ und er stellt die pointierte Frage: »Ist unser Land für seine Schriftsteller kein Gegenstand mehr? « ${ }^{8}$ Während Frischs eindringlich formulierte Frage auch als Aufruf an seine Landsleute verstanden werden kann, sich mit den blinden Flecken in der schweizerischen Geschichte auseinanderzusetzen, erhebt Reinacher keinen politischen Anspruch. Dennoch kann nicht über die Tatsache hinweggesehen werden, dass Reinachers ebenso wie Frischs Ausführungen am Ende einer Epoche stehen, in der die Schweiz eine tief greifende Krise erlebte, die ihr Selbstverständnis ins Wanken brachte.

In diesem Zusammenhang soll die Frage aufgeworfen werden, ob und inwieweit sich Pia Reinachers Darstellung der jungen Schweizer Schriftstellergeneration als Leitfaden eignet, um die neuere Literatur der Schweiz zu beleuchten. Insofern soll hier Reinachers These anhand dreier Schweizer Romane aus jüngeren Jahren auf die Probe gestellt werden, nämlich Zoë Jennys Das Blütenstaubzimmer (1997), Peter Stamms Agnes (1998) und Ruth Schweikerts Ohio (2005). Es geht darum zu untersuchen, ob und auf welche Weise die verschiedenen Diskurse über und um die Schweiz in diesen drei Werken explizit oder implizit angesprochen werden. Gegen Reinachers kritische Analyse möchte ich behaupten, dass sich die jüngeren Schweizer Schriftsteller sehr wohl mit ihrer Heimat auseinandersetzen, auch wenn sie dies auf verschlüsseltere Weise tun als ihre Vorgänger.

\section{Mythos Ferne}

Ruth Schweikerts 2005 erschienener Roman Ohio beschreibt, wie zwei der Protagonisten, Roberto und Amalia Lazzaroni, die Wände ihres Wohnzimmers mit

6 Walter Matthias Diggelmanns zuerst im Juli 1965 in der Zürcher Woche in Fortsetzungen veröffentlichte und dann im Oktober desselben Jahres als Buch publizierte Die Hinterlassenschaft muss hier als brisante Ausnahme gelten. W.M.D.: Die Hinterlassenschaft. Zürich: Edition 82003

7 Max Frisch: Unbewältigte schweizerische Vergangenheit. In: M.F.: Gesammelte Werke in zeitlicher Folge. Hg. von Hans Mayer unter Mitwirkung von Walter Schmitz. Bd. 5. Mein Name sei Gantenbein. Kleine Prosaschriften. Zürich-Transit. Biographie: Ein Spiel. Frankfurt/M.: Suhrkamp 1998, S. 370-373, hier S. 372.

8 Ebd., S. 373. Im März/April 1966 griff die Weltwoche Frischs provokative Frage auf und provozierte eine Flut von Reaktionen zum Thema, u.a. von Otto F. Walter, J.R. von Salis, Peter Bichsel und Adolf Muschg. Vgl. hierzu Klara Obermüller: Literatur der Zeitgenossenschaft. In: K.O. (Hg.): Wir sind eigenartig, ohne Zweifel. Die kritischen Texte von Schweizer Schriftstellern über ihr Land. München, Wien: Nagel \& Kimche 2003, S. 7-23, hier S. 13.

Please cite as: Valerie Heffernan, 'Unschweizerische Schweizerliteratuer? Ruth Schweikert, Peter Stamm, Zoë Jenny’, in: Schweiz schreiben. Zu Konstruktion und Dekonstruktion des Mythos Schweiz in der Gegenwartsliteratur, ed. by Jürgen Barkhoff and V. Heffernan, Tübingen: De Gruyter, 2010, pp. 283-295. 
Landkarten und Stadtplänen bedecken, die mit wenigen Ausnahmen Länder und Städte zeigen, die sie nie bereisten. ${ }^{9}$ Dieses Bild könnte auch als Metapher für die jüngere Schweizer Literatur fungieren, denn diese fokussiert sich sehr häufig auf entfernte Orte, die möglichst weit weg von dem Herkunftsland ihrer Verfasser liegen. Dies könnte als Anzeichen für Reinachers Befund der Schweiz-Flucht zeitgenössischer Autoren gelten. Denn betrachtet man die Texte Peter Stamms, Zoë Jennys und Ruth Schweikerts, kann man in der Tat verstehen, warum Reinacher beteuert, dass sie sich absichtlich von der helvetischen Heimat distanzieren. Es ist tatsächlich auffällig, wie diese drei Autoren den expliziten Bezug auf die Schweiz aussparen und ihre Geschichten entweder ganz oder zumindest zum Teil im Ausland ansiedeln. Die Erzählungen werden in Chicago, Durban, Paris, Berlin, Skandinavien, London oder Lissabon angesiedelt, oder deren Hauptfiguren träumen von einem besseren Leben in der Ferne. Wenn die Schweiz überhaupt vorkommt, so erscheint sie oft nur als Herkunftsort der Protagonisten oder als reine Kulisse ohne erkennbare thematische Bedeutung.

Jo, Zoë Jennys Protagonistin im Blütenstaubzimmer, sucht ihre Mutter in einem Land, das nie genannt wird, in dem aber die Sonne immer scheint, Feigenbäume wachsen und potentielle Liebhaber Luciano oder Vito heißen. Den Indizien im Text nach scheint das Land Italien zu sein, doch ist eine genaue Lokalisierung offenbar unwichtig; das einzig Wichtige ist, dass es anders als das Herkunftsland ist. Hier wird das Ausland als Paradies dargestellt, und Jo hofft endlich dort die Mängel in ihrem bisherigen Leben durch eine fruchtbare Beziehung zu ihrer Mutter auszugleichen. Sobald es jedoch scheint, als ob diese Hoffnung nicht in Erfüllung gehen wird, beginnt sich Jo nach einem Neuanfang an einem noch weiter entfernten Ort zu sehnen. Diesmal werden ihre Träume von einem besseren Leben geografisch verortet, und zwar in den Vereinigten Staaten; Milwaukee ist der Ort ihrer Träumereien. Diese amerikanische Stadt, die bei der Leserschaft womöglich Vorstellungen von Bierbrauereien und Fertigungsindustrie, technischer Entwicklung und Konsumgesellschaft hervorruft, bietet für Jennys Protagonistin die Möglichkeit eines neuen Lebens in der Anonymität, und das scheint den USA eigen zu sein.

Ebenso wählt Peter Stamm die USA für seine Erzählung Agnes, die Pia Reinacher als »alles andere als ein schweizerischer Roman « bezeichnet. ${ }^{10}$ Der Protagonist von Stamms Roman verlässt seine helvetische Heimat, um in der Weltstadt Chicago sein Glück zu suchen. Auf diese Weise nimmt der Autor Abstand von seiner Heimat in diesem Roman, wie eben auch in seinen anderen Werken. Bemerkenswert ist, dass sich Stamms Romane und Kurzgeschichten nicht nur inhaltlich von der Schweiz distanzieren; Kritiker weisen häufig auf die amerikanischen Vorbilder hin, die Stamms Schreibstil beeinflussen. Sie stellen häufig in Stamms Prosa die Fortsetzung amerikanischer Erzähltraditionen fest, wie die von Ernest Hemingway, Raymond Carver und Richard Ford. ${ }^{11}$

9 Ruth Schweikert: Ohio. Zürich: Ammann 2005, S.21.

10 Reinacher: Je Suisse (Anm. 1), S. 12.

11 Vgl. zum Beispiel Hubert Spiegel: Erdmännchenblicke. In: Frankfurter Allgemeine Zeitung, Nr. 42, 19.2.2000; Christine Lötscher: Die schwierige Freiheit der Fische. In: Tages-

Please cite as: Valerie Heffernan, 'Unschweizerische Schweizerliteratuer? Ruth Schweikert, Peter Stamm, Zoë Jenny’, in: Schweiz schreiben. Zu Konstruktion und Dekonstruktion des Mythos Schweiz in der Gegenwartsliteratur, ed. by Jürgen Barkhoff and V. Heffernan, Tübingen: De Gruyter, 2010, pp. 283-295. 
Ruth Schweikerts Ohio ist da allerdings nicht so leicht einzuordnen, weil der Roman die Chronik dreier Generationen der Familie Lazzaroni erzählt. Auf der einen Seite bildet die Schweiz den Schauplatz für die erinnerte Geschichte der ersten und zweiten Generationen, und das Geschehen auf dieser Erinnerungsebene spielt sich vorwiegend in der Schweiz ab. Auf der anderen Seite träumen die Protagonisten fortwährend von einem besseren Leben woanders, weg von der Schweiz und dem Ort ihrer gescheiterten Hoffnungen. So ziehen sich Andreas und Merete Lazzaroni in Meretes Geburtsort Durban in Südafrika zurück, um von dort aus eine neue Perspektive auf ihr Leben in der Schweiz und ihr Verhältnis zueinander zu gewinnen. Die Sehnsucht nach fremden und fernen Orten zeigt sich aber vor allem in den Tagträumen von Andreas' Großeltern: Roberto und Amalia, die italienischen Gastarbeiter, die sich in Celerina im Engadin niederlassen, kultivieren ihre Phantasien von einem entfernten Ort namens Ohio. Diese Sehnsucht nach einem neuen Leben in Ohio reichen sie auch unreflektiert weiter an ihren kleinen Sohn Michele, Andreas' Vater:

Als kleines Kind hatte er [Michele] - er konnte noch nicht richtig lesen, die Buchstaben waren Bilder für ihn - in die Rinden der Arvenbäume OHIO geritzt, das $\mathrm{H}$ war eine Leiter und das I eine Straße in die Zukunft, eingerahmt von den beiden O: die offenen Münder seiner Eltern Roberto und Amalia, wenn sie am späten Nachmittag auf dem Sofa dösten. ${ }^{12}$

Diese Zeilen enthüllen die Künstlichkeit und Inhaltslosigkeit der Vorstellung der USA, die der kleine Michele von seinen Eltern erwirbt; sie ist so einfach, dass sie auch kindlicher Fantasie zugänglich ist. Wie hier offensichtlich wird, hat das Amerika, das in den Texten neuerer Schriftstellerinnen und Schriftsteller wie Stamm, Jenny und Schweikert erscheint, wenig mit der Realität zu tun, sondern es erscheint immer wieder als Projektionsfläche für die Wünsche und Sehnsüchte der Figuren in den Texten.

Was Reinacher aber in ihrer Auffassung der Schweizer Gegenwartsliteratur übersieht, ist, dass die Tendenz der Gegenwartsliteratur, das Ausland zu thematisieren, keineswegs einen »Wandel« repräsentiert. Im Gegenteil, in der Schweizer Literaturgeschichte wurde die Häufigkeit der Auslandserfahrungen sowohl für die Roman-Protagonisten als auch für die Schriftsteller selbst verschiedentlich konstatiert. Paul Nizon sah dieses Phänomen eher aus negativer Perspektive; in seinem inzwischen bekannt gewordenen Essay Diskurs in der Enge von 1970 beschrieb er die »Enge« der Schweiz, die »den Künstler aus dem Lande oder in die voreilige Vergeistigung treibt «. ${ }^{13}$ Für ihn müssten die Schweizer Künstler ihrem kleinen Land entkommen und ins Ausland flüchten, um produktiv schreiben und leben zu können. Peter von Matt dagegen sieht die Auslandserfahrung etwas positiver an, nämlich als eine Art »doppelte Heimat « oder eine

Anzeiger, 25.8.2001; Ulrich Rüdenauer: Ein Hemingway après al lettre. In: Frankfurter Rundschau, 17.12.2003.

12 Schweikert: Ohio (Anm. 9), S. 124.

13 Paul Nizon: Diskurs in der Enge. In: P.N.: Diskurs in der Enge. Verweigerers Steckbrief. Schweizer Passagen. Hg. von Peter Henning. Frankfurt/M.: Suhrkamp 1990, S. 137-226, hier S. 167.

Please cite as: Valerie Heffernan, 'Unschweizerische Schweizerliteratuer? Ruth Schweikert, Peter Stamm, Zoë Jenny’, in: Schweiz schreiben. Zu Konstruktion und Dekonstruktion des Mythos Schweiz in der Gegenwartsliteratur, ed. by Jürgen Barkhoff and V. Heffernan, Tübingen: De Gruyter, 2010, pp. 283-295. 
»Zirkelexistenz: ein Bein in der Heimat, das andere im Ausland«.14 Von Matt unterstreicht, dass die Protagonisten wie auch die Autoren selbst sehr oft in die Heimat zurückkehren und das eigene Land nun mit anderen Augen ansehen. Auf diese Weise steht das Ausland beständig in einem engen Verhältnis zur Heimat.

Die Fokussierung auf die USA in zeitgenössischen Texten setzt gleichermaßen einen für die Schweizer Literatur sehr prägnanten literarischen Topos fort. Von Jürg Federspiels Museum des Hasses (1969) und Max Frischs Montauk (1975) über Hugo Loetschers Herbst in der Großen Orange (1982) und Franz Bönis Amerika (1992) bis zu Rolf Lapperts Amerikanische Trilogie (19942008) hat die USA in der Schweizer Literatur seit Langem eine signifikante Rolle gespielt ${ }^{15}$ - die Tradition wird in diesen drei Romanen fortgesetzt. Es ist wichtig zu bemerken, dass das Amerika der Schweizer Literatur fast ausschließlich als Bild, als klischeehaftes Image einen Wert hat. Die Schweizer Autoren lassen sich in ihren Werken bewusst auf die Klischees der USA ein und setzten sich oft kritisch oder ironisch mit den Stereotypen Amerikas auseinander, die durch Hollywoodfilme und Kriminalromane vermittelt werden. So werden die Vereinigten Staaten immer wieder idealisiert als Land der unbegrenzten Möglichkeiten oder als das Zentrum von Freiheit und Fortschritt. Ähnlich wie die deutschsprachige Literatur insgesamt, stellen auch die Schweizer Schriftsteller viel öfter die amerikanischen Großstädte in ihren Texten dar; vor allem die Wolkenkratzer New Yorks dienen sehr häufig als Hintergrund zu ihren Erzählungen. ${ }^{16}$

Doch ist es wichtig anzumerken, dass das Ausland, und vor allem das literarisierte Amerika, immer als Kontrast zur kleinen, konservativen, als restriktiv und eng wahrgenommen Schweiz fungiert. Romey Sabalius betont die Wichtigkeit der USA als Kontrastfolie zur begrenzten und kleinbürgerlichen Schweiz: »In dieser Hinsicht stellt sich der nordamerikanische Kontinent ganz besonders gegenüber der sprichwörtlichen Enge der Schweiz als Gegenstück par excellence dar, sowohl unter geographischen als auch gesellschaftlichen Gesichtspunkten. «17 Die mehrfache Wiederholung des Amerikabildes in der Schweizer Literatur und vor allem die vielen Klischees, mit denen dieses Bild durchdrungen ist, unterstreichen, dass das Bild mehr mit der Schweiz zu tun hat als mit der USA. Amerika bietet einen Ausweg aus der als eng wahrgenommenen Alpenheimat; nur in der Anonymität von Milwaukee oder hoch oben in Chicagos Wolkenkratzern könne man den Mängeln und der Leere des Schweizer Alltags

14 Peter von Matt: Zirkelexistenzen. Die doppelte Heimat der Schweizer Literatur. In: Beat Schläpfer (Hg.): Swiss, Made. Die Schweiz im Austausch mit der Welt. Zürich: Scheidegger \& Spiess 1998, S. 93-100, hier S. 93.

15 Eine ausführliche Analyse dieser und anderer Schweizer Amerika-Texte bietet Romey Sabalius: Das Bild der USA in der zeitgenössischen Literatur der deutschsprachigen Schweiz. In: R.S. (Hg.): Neue Perspektiven der deutschsprachigen Literatur in der Schweiz. Amsterdamer Beiträge zur neueren Germanistik 40 (1997), S. 11-30.

16 Ebd., S. 18f. Vgl. auch hierzu Sigrid Bauschinger: Mythos Manhattan. Die Faszination einer Stadt. In: S.B, Horst Denkler und Wilfried Malsch (Hg.): Amerika in der deutschen Literatur. Neue Welt - Nordamerika - USA. Stuttgart: Reclam 1975, S.382-397.

17 Sabalius: Das Bild der USA (Anm. 15), S.12.

Please cite as: Valerie Heffernan, 'Unschweizerische Schweizerliteratuer? Ruth Schweikert, Peter Stamm, Zoë Jenny’, in: Schweiz schreiben. Zu Konstruktion und Dekonstruktion des Mythos Schweiz in der Gegenwartsliteratur, ed. by Jürgen Barkhoff and V. Heffernan, Tübingen: De Gruyter, 2010, pp. 283-295. 
entkommen. Dies wird vor allem in Stamms Agnes deutlich artikuliert, wenn die Amerikanerin Louise den Schweizer Protagonisten mit der Bemerkung abfertigt: »Das Bild, das sich die Europäer von Amerika machen, hat mehr mit ihnen selbst zu tun als mit Amerika.«18

Das Amerikabild bei Stamm, Jenny und Schweikert erweist sich ebenfalls als klischeehaft und realitätsfern. Der Ich-Erzähler in Agnes bemüht sich zwar um ein Happyend für seine und Agnes' gemeinsame Geschichte, doch bleibt Stamms Protagonisten die Möglichkeit wirklicher Liebe fern. Amerika mag wohl den Ruf haben, das Land der unbegrenzten Möglichkeiten zu sein, aber die Realität ist wesentlich anders. Ähnlich geht es Roberto und Amalia Lazzaroni in Ohio: Das Ehepaar weiß zwar, dass es Robertos Bruder und seiner Familie in den USA nicht gut gegangen ist, doch scheint dies ihren Glauben an ein hoffnungsgebendes Ohio nicht zu erschüttern. Im Gegenteil, den können sie nur insofern aufrechterhalten, als dieser Ort immer ein Traum bleibt und nie zur Realität wird:

Ohio war, soweit Michele wusste, nichts Besonderes. Ein welliges Tafelland, hatte Roberto das Schweizerische Lexikon zitiert, zwei- bis dreihundert Meter über dem Meer; die Bevölkerung arbeitete in der Eisen- und Stahlindustrie, im Maschinen- und Apparatebau. Dennoch oder gerade deswegen hatten sie diese Sehnsucht in all den Jahren auf kleinster Flamme genährt. ${ }^{19}$

Michele ist offenbar bewusst, dass seine Vorstellungen von Ohio mit dem wahren Ort nicht zu vereinbaren sind, doch scheint es, als ob die Lazzaronis diesen Traum von Ohio brauchen, um sich mit ihrem Leben in Celerina im Engadin abzufinden. Dass sie ihre Auswanderungspläne immer wieder verschieben, liegt weder an ihren mangelhaften Englischkenntnissen noch an Amalias Schwangerschaft, sondern an der inneren Notwendigkeit, ihre Vorstellungen von Ohio aufrechtzuerhalten.

Auch Jennys Protagonistin scheint ihre Gedanken an Milwaukee zu brauchen; wenn ihre Freundin Rea die Möglichkeit einer Reise dorthin ganz ausschließt, so verwandelt sich Jos Wunschvision in eine Angstvision: »Die Wörter Rea und Milwaukee schrumpfen zu kleinen harten Angstkugeln. Ich bin voll mit diesen Kugeln, die mich von innen ausbeulen und verformen, so daß ich in alle Richtungen auseinanderzubrechen drohe.«20 Ihre Wahl von Milwaukee als Projektionsfläche für ihre Hoffnungen und Träume mag ebenso willkürlich wie grundlos zu sein, doch nach dem Verlust dieser Vorstellung wird die Protagonistin von Depressionen heimgesucht.

Oberflächlich scheint es, als ob sich die Texte zeitgenössischer Autorinnen und Autoren sehr wohl von der Schweizer Heimat abkehren und einen Ausweg im Ausland suchen. Die Flucht in die Ferne kann jedoch nicht als Abkehr von dem Thema `Schweiz^ verstanden werden, denn gerade das Ausland bietet eine Projektionsfläche für die Kritik an der Heimat. Die drei Texte, die hier analysiert

18 Peter Stamm: Agnes. München: Btb bei Goldmann 2000, S. 102.

19 Schweikert: Ohio (Anm. 9), S. 80.

20 Zoë Jenny: Das Blütenstaubzimmer. München: Btb bei Goldmann 1999, S. 124.

Please cite as: Valerie Heffernan, 'Unschweizerische Schweizerliteratuer? Ruth Schweikert, Peter Stamm, Zoë Jenny’, in: Schweiz schreiben. Zu Konstruktion und Dekonstruktion des Mythos Schweiz in der Gegenwartsliteratur, ed. by Jürgen Barkhoff and V. Heffernan, Tübingen: De Gruyter, 2010, pp. 283-295. 
werden, mögen zwar das Thema Schweiz nicht explizit aufgreifen, doch sowohl die Kontrastfolie >Ausland wie auch der Rückgriff auf spezifisch schweizerische literarische Vorbilder in ihren Texten weisen darauf hin, dass dieses Thema doch nicht gänzlich aus der jüngeren Schweizer Literatur verschwunden ist.

\section{Mythos Schneetod}

Dass sich die jungen Schriftsteller nicht ganz von ihren literarischen Vorvätern und deren Schweiz-Obsession abwenden, zeigt sich auch deutlich in ihren literarischen Verfahren; ihre Texte spielen mit als schweizerisch etablierten Vorbildern und Motivkomplexen und berufen sich auf Motive und Bilder, die bereits in früheren Schweizer Texten eine prominente Rolle spielten. Doch ist dies keine affirmative Fortsetzung Schweizer Traditionen; die junge Generation verwendet solche in der Schweizer Literatur bekannten Motive und Metaphern gerade um kritisches Licht auf das Schweizer Selbstbild zu werfen.

Das vielleicht offenkundigste Beispiel für einen solchen Motivkomplex ist das Sterben im Schnee. Das Motiv des Kältetods wird in allen drei der oben genannten literarischen Werke auf unterschiedliche Weise gestreift, findet seine in der zeitgenössischen Literatur eindrucksvollste Ausformung aber in der phantastischen Schlussszene von Peter Stamms Agnes. Als Agnes das Ende der für sie und über sie geschriebenen Geschichte liest, wird suggeriert, dass sie sich einem literarisierten Tod im Schnee hingibt. Der Erzähler beschreibt diese fiktive Szene wie folgt:

Dann kniete sie nieder, legte sich hin und drückte ihr Gesicht in den pulvrigen Schnee. Langsam gewann sie das Gefühl zurück, erst in den Füßen, in den Händen, dann in den Beinen und Armen, es breitete sich aus, wanderte durch ihre Schultern und ihren Unterleib zu ihrem Herzen, bis es ihren ganzen Körper durchdrang und es ihr schien, als liege sie glühend im Schnee, als müsse der Schnee unter ihr schmelzen. ${ }^{21}$

Diese starke Schlussszene von Stamms Romans ist von einigen Kritikern als Replik eines von Robert Walser entworfenen Todes im Schnee rezipiert worden. ${ }^{22}$ Walser hat nämlich seinen eigenen Tod im Schnee fast fünfzig Jahre im Voraus literarisch prophezeit, indem er in seinem 1907 verfassten Roman Geschwister Tanner das Auffinden eines toten Dichters im Schnee beschrieb. ${ }^{23}$ In diesem $\mathrm{Zu}-$ sammenhang ist es signifikant, dass Agnes ihren eigenen Tod im Roman selbst vorausgesehen hat. Als beide Protagonisten auf einer Wanderung auf einen kleinen Friedhof stoßen, bemerkt Agnes: »Stell dir vor, in wenigen Wochen

21 Ebd., S. 153.

22 Vgl. hierzu Norbert Staub: Rieselnde Wirklichkeiten. In: NZZ, Nr. 233, 8.10.1998; Peter Hamm: Der Tod der erzählten Frau. In: Focus, Nr. 47, 16.11.1998.

23 Robert Walser: Geschwister Tanner. In: R.W.: Sämtliche Werke in Einzelausgaben. Hg. von Jochen Greven. Bd. 9. Frankfurt/M.: Suhrkamp 1986, S. $129 f$.

Please cite as: Valerie Heffernan, 'Unschweizerische Schweizerliteratuer? Ruth Schweikert, Peter Stamm, Zoë Jenny’, in: Schweiz schreiben. Zu Konstruktion und Dekonstruktion des Mythos Schweiz in der Gegenwartsliteratur, ed. by Jürgen Barkhoff and V. Heffernan, Tübingen: De Gruyter, 2010, pp. 283-295. 
liegt hier Schnee, und dann kommt für Monate niemand hierher, und alles ist ganz still und verlassen. Es heißt, zu erfrieren sei ein schöner Tod.«24

Peter von Matt datiert das Motiv des Kältetods noch weiter in der Vergangenheit, und betont, »daß sich das Sterben im Schnee, der Kältetod, seit dem 19. Jahrhundert als ein unheimlich-inständiges Motiv durch die Schweizer Literatur zieht. «25 Als exemplarische Autoren nennt er Jeremias Gotthelf, Gottfried Keller, Meinrad Inglin und Friedrich Dürrenmatt. Auch wenn sich Peter Stamm zu amerikanischen Vorbildern bekennt, lässt er sich motivgeschichtlich ebenso als Repräsentant einer spezifisch schweizerischen Tradition einordnen. Allerdings weicht seine Version des Kältetods signifikant von dem durch die Schweizer Literatur überlieferten Motiv ab. Es ist merkwürdig, wie Agnes' imaginärer Tod im oben zitierten Abschnitt nicht mit Kälte verbunden wird, sondern mit Hitze; indem sich Agnes hier einem keinesfalls unschweizerisch anmutenden Tod im Schnee hingibt, strahlt sie vor Wärme.

Bemerkenswert ist, dass auch Schweikert und Jenny mit Varianten dieses Motivs spielen. In Ruth Schweikerts Ohio erlebt Sarah, eine gute Freundin der zwei Protagonisten, einen langsamen Tod im Schnee, als sie bei einer Wanderung in den Bergen durch eine Gletscherspalte fällt und dort erfriert. »In einer Tiefe von ungefähr zwanzig Metern eingeklemmt zwischen den Eiswänden einer Gletscherspalte, konnte sie sich weder vorwärts noch rückwärts bewegen.«26 Auf diese Weise wird dem für die Schweizer Literatur sehr häufigen Motiv des Kältetods ein tieferer Sinn verliehen. Schweikert spielt hier auf die Begrenztheit an, die mit der Schweiz assoziiert ist und die spätestens seit Nizons provokativen Behauptungen der schweizerischen »Enge« eine wichtige Metapher für die Schweiz bildet. Nizons Konzept hat die Diskussionen über die Schweizer Literatur so sehr gefärbt, dass es zwischenzeitlich auch zum Klischee geworden ist. Insofern kann es kein Zufall sein, dass Ruth Schweikerts Version des Kältetods gerade diesen Aspekt der Schweiz auf unübersehbare Weise hervorhebt. Allerdings verstößt Schweikerts Variante des Motivs gegen die herkömmliche Vorstellung der »Enge«. Erstens signalisiert der tödliche Unfall nicht Erstarrung sondern Erneuerung und Veränderung; nach Sarahs Tod und dem gemeinsam erlebten Trauma zieht Andreas zu Merete und sie beginnen einen neuen Lebensabschnitt als Paar. Zweitens stellt Schweikert mit ihren Roman Nizons These grundsätzlich infrage. Indem die Autorin eine Erzählung produziert, die in der Schweiz angesiedelt ist, bekannte Schweizer Motive miteinbezieht und auf bekannte Topoi der Diskussionen über die Schweizer Kunst anspielt, beweist sie, dass man weder physisch noch literarisch aus der Alpenheimat flüchten muss, um produktiv schreiben zu können.

Die Unmöglichkeit, sich weder vorwärts noch rückwärts zu bewegen, erfährt auch Jo in Jennys Blütenstaubzimmer in schmerzlicher Weise, als sie nach dem

24 Stamm: Agnes (Anm. 18), S. 78.

25 Peter von Matt: Schreiben und Sterben einer Autorin: Adelheid Duvanel. In: P.v.M.: Die tintenblauen Eidgenossen. Über die literarische und politische Schweiz. München, Wien: Carl Hanser 2001, S. 298-310, hier S. 306. Vgl. auch P.v.M.: Zirkelexistenzen (Anm. 14), S. 96.

26 Schweikert: Ohio (Anm. 9), S. 154.

Please cite as: Valerie Heffernan, 'Unschweizerische Schweizerliteratuer? Ruth Schweikert, Peter Stamm, Zoë Jenny’, in: Schweiz schreiben. Zu Konstruktion und Dekonstruktion des Mythos Schweiz in der Gegenwartsliteratur, ed. by Jürgen Barkhoff and V. Heffernan, Tübingen: De Gruyter, 2010, pp. 283-295. 
gescheiterten Besuch bei ihrer Mutter wieder nach Hause kommt und alles verändert vorfindet. Der Vater, der einst das bunte Leben eines Bohemiens führte, wohnt jetzt in einem netten Vorort, fährt ein richtiges Familienauto und hat das Rauchen aufgegeben. Jo kann sich mit der neuen Realität nicht abfinden und verlässt das Haus in der frühen Morgendämmerung. Hoffnungs- und aussichtslos zieht sie sich in den Park zurück, wo es zu schneien beginnt:

Zwei alte Frauen sitzen wie ausgestopft dicht aneinandergedrängt auf einer Parkbank. [...] Ich weiß, ich störe sie. Aber ich bleibe trotzdem hier. Und sage ihnen nicht, daß ich zusehen will, wie der Schnee auf den Boden fällt. Solcher, der nicht haften bleibt und eine dicke weiche Schicht bildet, sondern schmilzt, und daß ich deshalb immer auf die nächste Flocke warte, auf den sekundenschnellen Augenblick, in dem sie auftrifft und noch nicht geschmolzen ist. Und gemeinsam mit ihnen hier warten werde, auf die weiße Schicht über dem Boden. Auf die Decke aus Schnee. ${ }^{27}$

Bei dieser Schlussszene von Jennys Roman kommt es zwar nicht zum Tod im Schnee, aber die Tradition, auf die die junge Autorin sich bezieht, ist deutlich. Die Vergänglichkeit des Lebens wird hier durch die Vergänglichkeit des Schnees abgebildet. Der Schnee, der in der Schweizer Literatur oft mit Stille und Frieden, aber auch manchmal mit Härte und Beständigkeit verbunden wird, wird bald schmelzen.

Die Themen und Motive, die in den Texten der jungen Schriftstellergeneration vorkommen, scheinen auf den ersten Blick einen Wandel in der literarischen Landschaft anzuzeigen. Vor allem die Tatsache, dass diese Autorinnen und Autoren ihre Erzählungen außerhalb der Schweiz ansiedeln, hinterlässt den Eindruck, dass es hier um eine Ablehnung der Heimat und ihrer literarischen Traditionen geht. Doch wenn man näher hinschaut, so enthüllen die Texte ihre Verwandtschaft mit früheren Schweizer Texten und ihre indirekte Auseinandersetzung mit der Schweiz. Dass alle drei Autoren bewusst und absichtlich Motive der Schweizer Literaturgeschichte wie das des Kältetods aufgreifen, bedeutet, dass sie sich in eine Tradition stellen und nicht, dass sie mit dieser Tradition brechen. Ihr Verhältnis zur Tradition erweist sich allerdings als komplex und ambivalent und nicht als affirmativ. Das Motiv scheint in der Schweizer Gegenwartsliteratur alles andere als eingefroren zu sein, sondern erweist sich als taufrisch und wird durch Stamm, Schweikert und Jenny neu belebt.

\section{Mythos Schweiz}

Wenn Reinachers Thesen zur Lage der zeitgenössischen Schweizer Literatur bei Stamm und Jenny problematisch erscheinen, dann scheinen sie bei Schweikerts Roman, der zwei Jahre nach Reinachers Analyse entstand, gar unzutreffend zu sein. Vor allem in dem Roman Ohio ist die Schweiz durchaus Gegenstand einer kritischen Auseinandersetzung. Bemerkenswert dabei ist, dass so viele der poli-

27 Jenny: Das Blütenstaubzimmer (Anm. 20), S. 139.

Please cite as: Valerie Heffernan, 'Unschweizerische Schweizerliteratuer? Ruth Schweikert, Peter Stamm, Zoë Jenny’, in: Schweiz schreiben. Zu Konstruktion und Dekonstruktion des Mythos Schweiz in der Gegenwartsliteratur, ed. by Jürgen Barkhoff and V. Heffernan, Tübingen: De Gruyter, 2010, pp. 283-295. 
tischen, wirtschaftlichen und gesellschaftlichen Krisen, die in den 1980er und 1990er Jahren das Selbstverständnis der Schweizer im Inland sowie das Bild der Schweiz im Ausland ins Wanken gebracht haben, thematisiert und kritisch reflektiert werden.

Schweikerts Erzählung fokussiert auf Andreas und Merete Lazzaroni, deren Beziehung langsam auseinandergeht und die sich die Frage stellen, wie und wodurch dieser Prozess angefangen hat. Ihre Beziehung ist allerdings mit anderen Beziehungen verquickt, mit der Beziehung von Andreas' Eltern, zum Beispiel, zwischen dem Vater, der der Sohn italienischer Gastarbeiter ist, und der Mutter, die als Mädchen aus dem Osten vertrieben wurde. Ebenso weist die Geschichte noch weiter in die Vergangenheit zurück, auf Andreas' Großeltern, die 1920 ihre Heimatdörfer in Italien verließen, um in einer Schweizer Kleinstadt Arbeit zu suchen. In Form einer Familiengeschichte schreibt Schweikert also auch zugleich ein Stück Schweizer Geschichte.

So kommen wichtige Ereignisse für die Dekonstruktion und Rekonstruktion des schweizerischen Selbstverständnisses auch in Schweikerts Ohio vor und tragen zu einem relativierten Bild der Schweiz bei. Merete hatte sich den Jugendunruhen in Zürich in den frühen 80er Jahren angeschlossen, die der Unzufriedenheit mit den herrschenden sozialen und politischen Verhältnissen unter der jüngeren Generation Ausdruck gaben. Für Merete, wie für viele andere Teenager aus wohlhabendem Hause, boten die Jugendgruppen die Möglichkeit, die Selbstgefälligkeit der Elterngeneration infrage zu stellen. ${ }^{28}$ Die Bewegungen in den Schweizer Städten um diese Zeit enthüllten erhebliche Risse im idyllischen Heile-Welt-Bild der Schweiz. Dass die Autorin gerade diese hervorhebt, und zwar recht früh im Roman, bezeugt gleich von Anfang an ihre Auseinandersetzung mit den politischen, sozialen und kulturellen Realitäten ihrer Heimat.

Auch das Klischee einer neutralen und humanitären Schweiz, die vom Zweiten Weltkrieg verschont blieb, ein Bild, das allmählich seit 1945, aber vor allem in den vielen Debatten in den 1990er Jahren, wesentlich revidiert wurde, wird in Schweikerts Roman kritisch reflektiert. Dies zeigt vor allem Almuts Vorstellung über die Schweiz. Almut, die in der unmittelbaren Nachkriegszeit mit ihrer Mutter und ihrer älteren Schwester aus Breslau nach Waldshut an der süddeutschen Grenze umgezogen ist und das zweifache Trauma von Vergewaltigung einerseits und dem Tod der kleinen Schwester andererseits schwer überwinden kann, nimmt die Schweiz von jenseits der Grenze als eine ungestörte Idylle wahr:

Von den beiden Dachkammern aus, die sie zu dritt bewohnten, Margarethe, Almut und die Mutter, sah man auf die andere Seite des Flusses. Dort lag die Schweiz mit ihren Seen, Feldern und Wäldern und den kleinen Städtchen dazwischen, die so hübsch und so intakt waren, daß sie sich wie die Kulisse zu einem Theaterstück ausnahmen, das seit Jahrhunderten ununterbrochen gespielt wurde. ${ }^{29}$

28 Schweikert: Ohio (Anm. 9), S. 10f.

29 Ebd., S. $45 f$.

Please cite as: Valerie Heffernan, 'Unschweizerische Schweizerliteratuer? Ruth Schweikert, Peter Stamm, Zoë Jenny’, in: Schweiz schreiben. Zu Konstruktion und Dekonstruktion des Mythos Schweiz in der Gegenwartsliteratur, ed. by Jürgen Barkhoff and V. Heffernan, Tübingen: De Gruyter, 2010, pp. 283-295. 
Almuts Vorstellungen von der Schweiz als heile Welt mögen dem Leser heute naiv vorkommen, doch hängen diese eng mit der Epoche zusammen. Von außen gesehen scheint die Schweiz 1953 ein friedliches, vom Krieg verschontes Land zu sein, vor allem im Vergleich zu der Desorientierung, der Zerstörung und der ungewissen Zukunft, die das Deutschland dieser Zeit charakterisieren. In ihrem Schreiben unterstreicht Schweikert allerdings gerade die Künstlichkeit dieses Images; die Schweiz erscheint so hübsch und so perfekt, dass sie Almut wie die »Kulisse zu einem Theaterstück« vorkommt. Auch wenn Almut ihre Sehnsüchte und Hoffnungen auf die Schweiz projiziert, so ist sie sich trotzdem bewusst, dass ihr idyllisches Bild der Schweiz ein inszeniertes ist, das nicht unbedingt mit der Realität zu vereinbaren ist.

Das Leben in der Schweiz erweist sich denn auch als alles andere als idyllisch, es gibt tatsächlich eine große Diskrepanz zwischen dem Bild und der Realität. Die Heirat mit dem hübschen Michele ist für beide ein Kompromiss, das glückliche gemeinsame Familienleben eine Fassade, hinter der sich Micheles frustrierte Träume und Almuts unbewältigte Traumata verbergen. Trotzdem ist diese Realität nicht nur negativ zu betrachten. Wie Almut nach dem Tod ihres Mannes betont: " >Ich weiß, daß mein Mann schwul war - sie sagte schwwuul, als bereite es ihr ein besonderes Vergnügen - strotzdem haben wir eine glückliche Ehe geführt. « ${ }^{30}$ Almut ist sich bewusst, dass ihr Verhältnis zu ihrem Mann kein perfektes war, aber sie akzeptierte ihn so, wie er war, und unter diesen Umständen konnten sie glücklich zusammen leben. Es muss kein perfektes Bild sein, so die Botschaft des Romans; mit relativierten und konsensfähigen Bildern, mit Kompromissen und Teillösungen könne man leben.

Das schweizerische Selbstbild als Musterland wirtschaftlichen Erfolgs und internationaler Solidarität ist in jüngeren Jahren auch durch das Scheitern des Schweizer Konzerns Swissair ins Wanken gekommen, und das ist eine weitere Krise, die im Roman thematisiert wird. Die nationale Fluggesellschaft, die lange Zeit das Schweizer Selbstverständnis mit großem Erfolg verkörperte, geriet im September 2001 in so große finanzielle Schwierigkeiten, dass die Maschinen am Boden blieben. Im Roman wird dieses Ereignis zum Symbol für das Zerbröckeln eines Schweizer Mythos aufgewertet. Andreas beschreibt die ohnmächtigen Flugzeuge, die nicht mehr fliegen dürfen, als »kleine dumme Spatzen, [...], die sich für Adler gehalten, sich überfressen und aufgebläht hatten, bis sie endlich platzten«. ${ }^{31}$ Und seine Haltung gegenüber dem Untergang der Swissair spiegelt die Reaktion der Mehrheit der Schweizer Bevölkerung wider, »ihre Bestürzung, ihre plötzliche Wut auf den Staat, die Banken und die verantwortlichen Manager«.32 Was bleibt von dem Bild einer effizienten, luxuriösen und zuverlässigen Fluggesellschaft, die in aller Welt als Bild der Schweiz gelten wollte? »Postkarten und Schlüsselanhänger, Bastelbögen und handliche Swissairflugzeuge aus Leichtmetall, Plüsch oder Plastik.«33 Diese

30 Ebd, S. $142 f$.

31 Ebd., S. 54.

32 Ebd.

33 Ebd.

Please cite as: Valerie Heffernan, 'Unschweizerische Schweizerliteratuer? Ruth Schweikert, Peter Stamm, Zoë Jenny’, in: Schweiz schreiben. Zu Konstruktion und Dekonstruktion des Mythos Schweiz in der Gegenwartsliteratur, ed. by Jürgen Barkhoff and V. Heffernan, Tübingen: De Gruyter, 2010, pp. 283-295. 
kitschigen Spielzeuge deuten nicht mehr stolz auf die Stärke eines Nationalkonzerns, sondern metonymisch auf die Abwesenheit der Swissair und somit eines einheitlichen, nationalen Selbstverständnisses hin. Sie verdinglichen somit das Bild der Schweiz, das die Swissair repräsentierte: Es war und ist ein Klischee.

Es sind aber nicht nur schweizerische Traumata, die in Ohio vorkommen, sondern auch auswärtige. Wichtig ist, dass die Schweiz in Schweikerts Roman nicht als autonome Insel dargestellt wird, sondern als ein Land, das durchaus in europäische und globale Verhältnisse verwickelt ist. So hätten die Attentate auf die Twin Towers in New York auch für die Schweiz eine Relevanz, und ebenso müsse man auf Besuch in Südafrika bedenken, dass Schweizer Banken während der Zeit der Apartheid die südafrikanische Regierung finanziell unterstützten. ${ }^{34}$ Was der Schweiz passiert, trifft auch das Ausland, und gleichermaßen führen globale Änderungen zu Wandlungen des Schweiz-Bildes.

Überhaupt regt dieser Roman zum Nachdenken über Bilder an, und das Bild der Schweiz, das dabei entsteht, erweist sich als ein wesentlich differenziertes. In Schweikerts Ohio, so meine These, werden die Risse und Narben, die die Revisionen des gängigen Schweiz-Bildes in den letzten Jahren hinterlassen haben, zum sichtbaren Teil des Bildes selbst. Zwei verschiedene bildliche Motive werden in den Text eingeflochten, welche darauf hinweisen, wie sich das Image der Schweiz im Laufe der letzten Jahrzehnte geändert hat.

Erstens werden Micheles merkwürdige Fotografien beschrieben. Michele gefällt es nämlich, mit Bildern zu spielen. Er macht gerne Fotos von Menschen, inszenierte Fotos, in denen sie mit geschlossenen Augen aufgenommen werden: "»Damit Sie sich selber sehen können, ‘ pflegte er ihnen zu sagen, >wie sonst nur andere Sie sehen.«35 Diese künstlichen Bilder, in denen der Betrachtete auch zum Betrachter wird, sagen viel aus über die Funktion der Blicke und der Bilder im Roman. Der Betrachter hat die Möglichkeit, aus der Distanz ein erstarrtes Bild von sich selbst in einer Position der Machtlosigkeit zu betrachten. Durch einen merkwürdig doppelten Blick gewinnt der Betrachter Einsicht in sich selbst, er sieht das, was er normalerweise nicht sehen kann. So fungiert diese photographische Praxis als Metapher für die Selbstanalyse.

Die Relevanz dieses Vorgangs für die Präsentation der Schweiz im Roman ist unübersehbar. Ruth Schweikerts Ohio kann als Porträt der heutigen Schweiz verstanden werden, und dies ist ebenfalls ein Bild der Schweiz mit geschlossenen Augen, wie sonst nur andere sie sehen. Es ist der Blick von außen, der klarsichtiger ist. Ähnlich wie Micheles eigenartige Fotografien stellt Schweikerts Porträt ihrer Heimat die Narben und Risse der jüngeren Vergangenheit bloß und veranschaulicht dabei die Zerbrechlichkeit und Schwäche der Schweiz.

Das zweite bildliche Motiv, das im Roman Verwendung findet, hängt mit Meretes Leidenschaft für Paul Klees zerschnittene Bilder, über die sie auch ihre Dissertation schreibt, zusammen. Klee hat nämlich mehr als dreihundert seiner Bilder in kleinere Teile zerschnitten und diese wieder neu zusammengefügt und

34 Vgl. hierzu S. 54, S. 58.

35 Ebd., S. 62.

Please cite as: Valerie Heffernan, 'Unschweizerische Schweizerliteratuer? Ruth Schweikert, Peter Stamm, Zoë Jenny’, in: Schweiz schreiben. Zu Konstruktion und Dekonstruktion des Mythos Schweiz in der Gegenwartsliteratur, ed. by Jürgen Barkhoff and V. Heffernan, Tübingen: De Gruyter, 2010, pp. 283-295. 
als eigenständige Bilder ausgestellt. Ähnlich wie Klees Bilder, mit denen Merete arbeitet, zerschneidet und dekonstruiert dieser Roman das geschönte Image einer heilen Schweiz, wie es bis weit nach 1945 gepflegt wurde; Teile davon werden neu zusammengestellt und konfiguriert, sodass ein wesentlich differenzierteres oder auch fragmentierteres Image entsteht. Das neue Image der Schweiz bezieht Bruchstücke der vielen klischeehaften Repräsentationen der Schweiz mit ein, aber es stellt eben auch die Künstlichkeit derselben bloß. In diesem Prozess entsteht ein ganz neues Image der Schweiz, in dem allerdings das alte sichtbar bleibt.

Ruth Schweikerts Ohio regt zum Nachdenken über das klischeehafte Bild der Schweiz an, das demontiert werden musste, damit das Land die Fehler der Vergangenheit konfrontieren und sie anerkennen konnte. Gleichzeitig konstruiert der Roman ein neues Bild der Schweiz, das zweifellos von den vielen Krisen und Skandalen der 1980er und 90er Jahre geprägt ist und die Spuren eines signifikanten Umdenkensprozesses trägt. Die Schweiz ist nicht das, was sie einmal schien, heißt es in diesem Roman, und Schweikert scheut sich nicht davor, sich zu diesem revidierten Bild ihrer Heimat zu bekennen.

Betrachtet man die Romane Peter Stamms, Zoë Jennys und Ruth Schweikerts näher, so kann man Pia Reinachers Diagnose eines »tief greifenden Wandel[s]« in der jüngsten Generation der Schweizer Literatur schlicht nicht nachvollziehen, denn diese Autoren kehren ihrer helvetischen Heimat nur oberflächlich den Rücken. Ihre Literatur mag wohl indirekter mit dem Thema `Schweiz` umzugehen als die eines Max Frisch oder eines Adolf Muschg oder sogar eines Thomas Hürlimann, doch bedeutet dies nicht, dass man ganz und gar von eine »neue[n] literarische[n] Identität« sprechen kann. ${ }^{36}$ Sie verwenden die Außenperspektive, um kritisches Licht auf ihre Heimat zu werfen und machen sich die literarischen Werkzeuge ihrer Vorgänger zunutze, um diese neu zu beleben. Durch ihre Literatur schreiben sie an und über die Schweiz - und sie schreiben sie neu.

36 Reinacher: Je Suisse (Anm. 1), S. 21.

Please cite as: Valerie Heffernan, 'Unschweizerische Schweizerliteratuer? Ruth Schweikert, Peter Stamm, Zoë Jenny’, in: Schweiz schreiben. Zu Konstruktion und Dekonstruktion des Mythos Schweiz in der Gegenwartsliteratur, ed. by Jürgen Barkhoff and V. Heffernan, Tübingen: De Gruyter, 2010, pp. 283-295. 
Please cite as: Valerie Heffernan, 'Unschweizerische Schweizerliteratuer? Ruth Schweikert, Peter Stamm, Zoë Jenny’, in: Schweiz schreiben. Zu Konstruktion und Dekonstruktion des Mythos Schweiz in der Gegenwartsliteratur, ed. by Jürgen Barkhoff and V. Heffernan, Tübingen: De Gruyter, 2010, pp. 283-295. 